Quim. Nova, Vol. 36, No. 7, 998-1003, 2013

\title{
EMISSÕES DE ÓXIDO NITROSO DO TANQUE DE AERAÇÃO DE UMA ESTAÇÃO DE TRATAMENTO DE ESGOTOS COM SISTEMA DE LODOS ATIVADOS CONVENCIONAL
}

\author{
Renato P. Ribeiro, William Z. de Mello*, Renata B. Alvim, Samara A. Andrade e Ariane C. Brotto \\ Departamento de Geoquímica, Instituto de Química, Universidade Federal Fluminense, 24020-141 Niterói - RJ, Brasil \\ Débora C. Kligerman e Jaime L. M. Oliveira \\ Departamento de Saneamento e Saúde Ambiental, Escola Nacional de Saúde Pública, Fundação Oswaldo Cruz, 21041-210 Rio \\ de Janeiro - RJ, Brasil \\ Paulo A. de Almeida \\ Companhia Estadual de Águas e Esgotos, CEDAE, Avenida Presidente Vargas, 2655, 20210-031 Rio de Janeiro - RJ, Brasil
}

Recebido em 26/10/12; aceito em 24/3/13; publicado na web em 14/5/13

\begin{abstract}
NITROUS OXIDE EMISSIONS FROM THE AERATION TANK OF A WASTEWATER TREATMENT PLANT WITH CONVENTIONAL ACTIVATED SLUDGE PROCESS. Nitrous oxide $\left(\mathrm{N}_{2} \mathrm{O}\right)$ emissions were measured monthly from January to June 2010 in the aeration tank of a wastewater treatment plant (WWTP) in Southeast Brazil. Emissions were lower in summer than winter and were positively related with influent ammonium $\left(\mathrm{NH}_{4}^{+}\right)$concentration. The average $\mathrm{N}_{2} \mathrm{O}$ emission was $1.11 \mathrm{~kg} \mathrm{~N}^{-1}$ corresponding to $0.02 \%$ of the influent total nitrogen load. The average emission factor calculated for the population served was 2.5 lower than that proposed by the Intergovernmental Panel on Climate Change (IPCC) for inventories of $\mathrm{N}_{2} \mathrm{O}$ emissions from WWTPs with controlled nitrification and denitrification processes.
\end{abstract}

Keywords: nitrous oxide; wastewater treatment plant; activated sludge.

\section{INTRODUÇÃO}

$\mathrm{O}$ óxido nitroso $\left(\mathrm{N}_{2} \mathrm{O}\right)$ possui grande importância ambiental em função do papel que desempenha na química e física da atmosfera. $\mathrm{Na}$ troposfera, é um dos principais gases do efeito estufa e possui potencial de aquecimento 300 e 15 vezes superior ao do dióxido de carbono $\left(\mathrm{CO}_{2}\right)$ e metano $\left(\mathrm{CH}_{4}\right)$, respectivamente. ${ }^{1} \mathrm{O}$ principal sumidouro do $\mathrm{N}_{2} \mathrm{O}$ é na estratosfera, onde uma parcela equivalente a $5 \%$ dele é convertida a óxido nítrico (NO) através das reações com átomos de oxigênio excitado. ${ }^{2} \mathrm{O}$ NO é um dos principais gases responsáveis pelo consumo do ozônio $\left(\mathrm{O}_{3}\right)$ estratosférico, ${ }^{3}$ e o $\mathrm{N}_{2} \mathrm{O}$ se tornará a principal substância emitida por atividades humanas que mais danos causará a camada de $\mathrm{O}_{3}$ ao longo do século XXI. ${ }^{4}$

Nos últimos 30 anos, as concentrações de $\mathrm{N}_{2} \mathrm{O}$ na atmosfera superficial vêm crescendo linearmente na ordem de $0,25 \%$ ao ano. ${ }^{5} \mathrm{Em}$ 2006 , cerca de $45 \%$ do total global de $\mathrm{N}_{2} \mathrm{O}$ emitido para a atmosfera foi originado de fontes antrópicas, sendo a agricultura responsável por aproximadamente $2 / 3$ do total dessas emissões. ${ }^{6}$ Atualmente sua concentração atmosférica é aproximadamente $325 \mathrm{ppb},{ }^{7}$ o que representa um aumento de $20 \%$ se comparado ao período pré-industrial. ${ }^{5,8}$ Outra fonte antrópica deste gás são os processos de tratamento de esgotos, cuja contribuição é quantitativamente considerada de menor relevância. ${ }^{9,10}$ Entretanto, pouco se sabe sobre a real contribuição dessas emissões em virtude da grande variabilidade observada nos fatores de emissão, balizados na carga de nitrogênio total (NT) afluente, disponíveis na literatura oriundos de estudos realizados tanto em escala real $(0-25 \%)^{11,12}$ quanto laboratorial (0-95\%). ${ }^{12}$

Para inventários de emissão de $\mathrm{N}_{2} \mathrm{O}$, o Intergovernmental Panel on Climate Change (IPCC) propõe o uso do fator de emissão (FE) 3,2 $\mathrm{g} \mathrm{N}_{2} \mathrm{O}$ pessoa $^{-1}$ ano $^{-1}$ para Estações de Tratamento de Esgotos (ETEs) com processos de nitrificação e desnitrificação controlados, ${ }^{9}$ baseado

*e-mail: zamboni@geoq.uff.br

\#endereço atual: Department of Earth and Environmental Engineering, Columbia University, New York - NY 10027, USA no estudo realizado por Czepiel et al. ${ }^{13} \mathrm{em}$ uma ETE de lodos ativados localizada na cidade de Durham (EUA). Entretanto, a United States Environmental Protection Agency (US-EPA) adota o FE 7,0 g $\mathrm{N}_{2} \mathrm{O}$ pessoa $^{-1}$ ano $^{-1}$ para o caso de ETEs com processos de desnitrificação intencional, e o FE 3,2 $\mathrm{g} \mathrm{N}_{2} \mathrm{O}$ pessoa $^{-1}$ ano $^{-1}$ para ETEs com processos de desnitrificação não intencional. ${ }^{10}$

Basicamente, a produção de $\mathrm{N}_{2} \mathrm{O}$ em sistemas de tratamento de esgotos está associada aos processos microbiológicos de nitrificação e desnitrificação que ocorrem em ambientes naturais. ${ }^{11,14}$ No entanto, os mecanismos que conduzem à formação do $\mathrm{N}_{2} \mathrm{O}$ são ainda amplamente discutidos na literatura e oriundos de estudos realizados em sua maioria em escala laboratorial. ${ }^{12,15-17}$ No caso de ETEs com sistemas de lodos ativados, apesar de, em escala real, ainda não haver um completo entendimento dos mecanismos que levam à emissão de $\mathrm{N}_{2} \mathrm{O}$, Czepiel et al. ${ }^{13}$ reportaram que $91 \%$ do total das emissões de $\mathrm{N}_{2} \mathrm{O}$ de uma ETE de lodos ativados no nordeste dos Estados Unidos foram oriundos do tanque de aeração. Brotto et al. ${ }^{18}$ encontraram resultado semelhante em uma ETE de lodos ativados localizada no sudeste do Brasil. Em estudo realizado em 12 ETEs localizadas nos EUA, Ahn et al. ${ }^{19}$ indicaram que elevadas concentrações de amônio $\left(\mathrm{NH}_{4}^{+}\right)$, nitrito $\left(\mathrm{NO}_{2}{ }^{-}\right)$e oxigênio dissolvido (OD) no tanque de aeração estão positivamente correlacionados com as emissões de $\mathrm{N}_{2} \mathrm{O}$. Através de estudo realizado em escala piloto, Lotito et al. ${ }^{20}$ complementam que a conversão de $\mathrm{NH}_{4}{ }^{+}$a $\mathrm{N}_{2} \mathrm{O}$ está fortemente relacionada a parâmetros operacionais, tais como concentração de NT afluente, idade do lodo e concentração de OD.

O presente estudo teve como proposta a continuação de investigações que vêm sendo desenvolvidas no Brasil por nosso grupo de pesquisa em diferentes ETEs de lodos ativados. ${ }^{18,21,22}$ Tem como objetivo avaliar a relação entre alguns parâmetros operacionais, tais como concentração de NT afluente à ETE (especialmente $\mathrm{NH}_{4}^{+}$), concentração de demanda química de oxigênio (DQO) e OD, com as emissões de $\mathrm{N}_{2} \mathrm{O}$ do tanque de aeração de uma ETE que utiliza o sistema de lodos ativados convencional, com processo de aeração diferenciado por zonas. 


\section{PARTE EXPERIMENTAL}

\section{Área de estudo}

A ETE escolhida para a realização do presente estudo está localizada na região metropolitana do Rio de Janeiro. Essa ETE faz parte do Programa de Despoluição da Baía de Guanabara e foi projetada para atender a uma população de aproximadamente 1,25 milhões de habitantes - atualmente atende $c a .38 \%$ desse montante. É uma ETE considerada de grande porte e que utiliza como sistema de tratamento de esgotos o processo de lodos ativados convencional, com tempo de detenção hidráulica de aproximadamente 9 horas e idade do lodo variando de 10 a 12 dias. A vazão de esgoto bruto afluente à ETE durante o período de estudo variou de $5,6 \times 10^{3}$ a $6,8 \times 10^{3} \mathrm{~m}^{3} \mathrm{~h}^{-1}$.

A ETE possui como unidades de tratamento de esgotos o tratamento preliminar (composto por gradeamento, elevatória de esgoto bruto, medidor Parshall e desarenador), o tratamento primário (composto por decantador primário), o tratamento secundário (composto pelo tanque de aeração e o decantador secundário) e o tratamento do lodo (composto por adensador, digestor anaeróbio e sistema de desaguamento do lodo).

O processo de aeração nessa ETE é diferenciado por zonas, sendo a aeração realizada em um total de 6 zonas (ZNs) em cada um dos 4 tanques de aeração. A ZN1 é diferenciada das demais zonas pela presença de difusores de bolhas grossas, possuindo apenas a função de homogeneização do efluente do tratamento primário e do lodo biológico que retorna do decantador secundário. As demais zonas são caracterizadas por possuírem difusores de bolhas finas, tendo como vantagens boa capacidade de mistura e elevada transferência de oxigênio. ${ }^{23} \mathrm{~A}$ vazão de ar de cada zona é variada em virtude do número de difusores presentes em cada uma delas (Figura 1). Durante o período de estudo, a vazão total de ar nos 4 tanques de aeração (dos 8 que a ETE possui) estudados variou de $1,8 \times 10^{4}$ a $2,1 \times 10^{4} \mathrm{~m}^{3} \mathrm{~h}^{-1}$

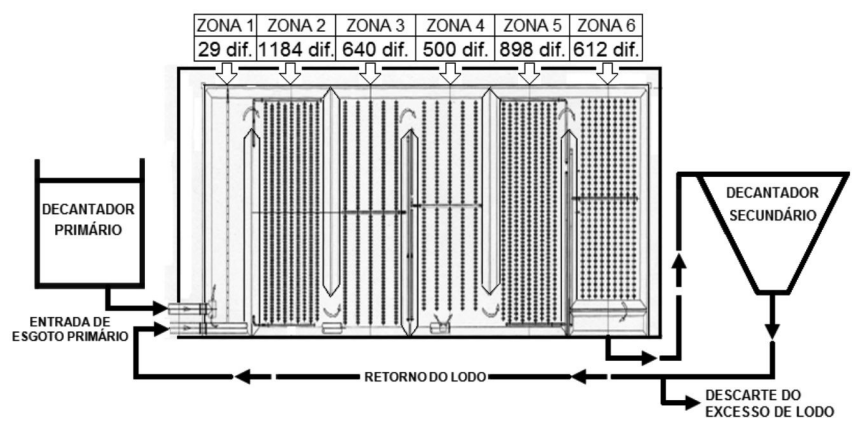

Figura 1. Representação esquemática de um dos quatro tanques (idênticos) de aeração que compõem a ETE de estudo, mostrando o número de difusores presentes em cada uma das seis zonas

\section{Amostragem e análises}

A amostragem foi realizada de forma a avaliar o comportamento e magnitude das emissões de $\mathrm{N}_{2} \mathrm{O}$ em função dos parâmetros operacionais, concentração de $\mathrm{NH}_{4}{ }^{+}$afluente à ETE e concentrações de DQO e OD em cada zona dos tanques de aeração. As amostragens ocorreram no período de janeiro a junho de 2010, uma vez por mês e sempre pela manhã, entre 08:00 e 11:00 h, totalizando 6 campanhas realizadas. Em cada dia de amostragem foram efetuadas medidas de fluxos de $\mathrm{N}_{2} \mathrm{O}$ em 10 pontos distribuídos aleatoriamente entre as zonas dos 4 tanques de aeração de forma que todas as zonas (ZN1 a ZN6) fossem amostradas.
Para a determinação do fluxo de $\mathrm{N}_{2} \mathrm{O}$ na interface esgoto-atmosfera em cada zona dos tanques de aeração foi utilizada como metodologia de amostragem a técnica do funil emborcado descrita detalhadamente por Brotto et al. ${ }^{18}$ Essa técnica permite a captação das bolhas de ar que se desprendem da superfície do esgoto aerado, com a finalidade de se determinar a concentração de $\mathrm{N}_{2} \mathrm{O}$ no ar desprendido do esgoto para posterior cálculo do fluxo do gás. Após imersão parcial do funil para coleta da amostra, aguardou-se pelo menos 3 minutos até que houvesse a estabilização da concentração do $\mathrm{N}_{2} \mathrm{O}$ no headspace do funil. Em cada um dos 10 pontos de amostragem foi coletada uma amostra de ar atmosférico e 4 amostras do interior do headspace do funil, totalizando 50 amostras para determinação de $\mathrm{N}_{2} \mathrm{O}$. A obtenção de 4 amostras por ponto de amostragem teve como objetivo verificar a homogeneidade da concentração de $\mathrm{N}_{2} \mathrm{O}$ no headspace do funil.

$\mathrm{O}$ fluxo (F) de $\mathrm{N}_{2} \mathrm{O}$ é calculado, segundo a equação (1), através do produto da vazão de ar injetado $(\mathrm{Q})$ em cada zona pela diferença entre a concentração média $(\mathrm{n}=4)$ de $\mathrm{N}_{2} \mathrm{O}$ no headspace do funil e a sua concentração na atmosfera $\left(\Delta \mathrm{N}_{2} \mathrm{O}\right)$, divida pela área $(\mathrm{A})$ de cada zona $\left(\mathrm{ZN} 1=181 \mathrm{~m}^{2}\right.$ e ZN2-6 = $\left.399 \mathrm{~m}^{2}\right)$ :

$$
\mathrm{F}=\left(\mathrm{Q} \times \Delta \mathrm{N}_{2} \mathrm{O}\right) / \mathrm{A}
$$

As amostras de ar foram analisadas no mesmo dia da amostragem (até 8 horas após seu início) através da utilização de um cromatógrafo a gás (Shimadzu, modelo GC-17A) equipado com coluna empacotada com Porapak-Q, mantida a $60^{\circ} \mathrm{C}$, e detector de captura de elétrons $\left({ }^{63} \mathrm{Ni}\right)$, operado a $340{ }^{\circ} \mathrm{C}$. O gás de arraste utilizado foi o argônio contendo 5\% de metano (White Martins Ltda), numa vazão de $40 \mathrm{~mL}$ $\min ^{-1}$. Foram utilizados para a construção da curva analítica padrões de 356 e 840 ppb (White Martins Ltda). Os limites de detecção e quantificação foram 30 e $300 \mathrm{ppb}$, respectivamente. A precisão analítica das determinações baseada nos valores de área obtidos para a concentração atmosférica de $\mathrm{N}_{2} \mathrm{O}$, realizada em triplicata, foi aproximadamente $\pm 5 \%$. Os coeficientes de variação das médias das 4 determinações de concentração de $\mathrm{N}_{2} \mathrm{O}$ no headspace do funil, de 60 medidas realizadas ( 6 campanhas $\times 10$ pontos de amostragem), foram inferiores a $6 \%$.

Durante as amostragens também foram realizadas medições in situ da concentração de OD e pH por meio de uma sonda multiparamétrica (Hanna Instruments, modelo HI-9828). Foram coletadas amostras de esgoto bruto e tratado para a determinação das concentrações de nitrogênio inorgânico dissolvido (NID $=\mathrm{NH}_{4}^{+}+$ $\mathrm{NO}_{2}^{-}+\mathrm{NO}_{3}^{-}$) e NT, além de amostras do tanque de aeração para a determinação da DQO em laboratório. Os dados de concentração da demanda bioquímica de oxigênio (DBO) e DQO referentes ao esgoto bruto (afluente) e tratado (efluente) foram fornecidos pelo laboratório da ETE e as coletas realizadas três vezes por mês por operadores da ETE em dias diferentes às datas de amostragem.

As amostras para determinação de NID foram filtradas em membrana de acetato de celulose com diâmetro de poro de $0,22 \mu \mathrm{m}$ e armazenadas (congeladas) em freezer para posterior análise através dos métodos colorimétricos descritos no Standard Methods for the Examination of Water and Wastewater. ${ }^{24}$ Os limites de detecção e quantificação para $\mathrm{NH}_{4}^{+}$e nitrato $\left(\mathrm{NO}_{3}^{-}\right)$foram 0,02 e $0,2 \mathrm{mg} \mathrm{N} \mathrm{L}^{-1}$ e para $\mathrm{NO}_{2}^{-}$foram 0,002 e $0,02 \mathrm{mg} \mathrm{N} \mathrm{L}^{-1}$, respectivamente. As precisões analíticas das determinações de NID realizadas em triplicata estiveram na faixa de $\pm 5 \%$. As análises para determinação de NT e DQO foram realizadas em amostras não filtradas e acidificadas com ácido sulfúrico para $\mathrm{pH}$ próximo a 2,0. As concentrações de NT e DQO foram determinadas pelos métodos colorimétricos após digestão das amostras com soluções de persulfato de potássio e dicromato de potássio, respectivamente. ${ }^{24}$ 
Tabela 1. Fatores de emissão (FEs) médios balizados na vazão de esgoto bruto, população atendida e carga afluente de nitrogênio total deste estudo e outros realizados em ETEs de lodos ativados no Brasil

\begin{tabular}{|c|c|c|c|c|}
\hline Referência & Sistema de aeração empregado & $\mathrm{FE}\left(\mathrm{g} \mathrm{N}_{2} \mathrm{O} \mathrm{L}_{\text {esgoto }}{ }^{-1}\right)$ & $\mathrm{FE}\left(\mathrm{g} \mathrm{N}_{2} \mathrm{O}\right.$ pessoa $^{-1}$ ano $\left.^{-1}\right)$ & $\mathrm{FE}\left(\%\right.$ carga de $\left.\mathrm{NT}_{\text {afluente }}\right)$ \\
\hline Este estudo & Convencional & $1,2 \times 10^{-5}$ & 1,3 & 0,02 \\
\hline Brotto et al. ${ }^{18}$ & Prolongada & $9,0 \times 10^{-5}$ & 13 & 0,14 \\
\hline Brotto $^{21}$ & Modificada & $8,0 \times 10^{-5}$ & 8,1 & 0,12 \\
\hline de Mello et al..$^{22}$ & Intermitente & $8,0 \times 10^{-5}$ & 8,8 & 0,10 \\
\hline
\end{tabular}

\section{RESULTADOS E DISCUSSÃO}

\section{Características do esgoto bruto}

As médias aritméticas (desvio padrão) das concentrações de DBO e DQO no esgoto bruto foram $345 \mathrm{mg} \mathrm{L}^{-1}\left(166 \mathrm{mg} \mathrm{L}^{-1}\right)$ e $634 \mathrm{mg}$ $\mathrm{L}^{-1}\left(244 \mathrm{mg} \mathrm{L}^{-1}\right)$, respectivamente. Com base em Jordão e Pessôa, ${ }^{25}$ esses valores caracterizam o afluente da ETE estudada como esgoto doméstico com carga orgânica variando de média a forte. Em relação às concentrações de NT, a média (desvio padrão) determinada foi 43,0 $\mathrm{mg} \mathrm{L}^{-1}\left(8 \mathrm{mg} \mathrm{L}^{-1}\right)$, valor próximo à média reportada por Oliveira $\mathrm{e}$ von Sperling ${ }^{26}$ para ETEs com sistemas de lodos ativados. Durante o período de estudo o valor médio (desvio padrão) do $\mathrm{pH}$ foi $6,4(0,3)$, o que caracteriza praticamente toda a amônia $\left(\mathrm{NH}_{3}\right)$ na forma ionizada $\left(\mathrm{NH}_{4}^{+}\right){ }^{23} \mathrm{Em}$ média, $\mathrm{o} \mathrm{NH}_{4}{ }^{+}$correspondeu a aproximadamente $60 \%$ do NT. O restante é atribuído ao nitrogênio orgânico total (e.g. uréia, aminoácidos, proteínas, etc.), determinado por diferença entre o NT e NID, que posteriormente é degradado a $\mathrm{NH}_{4}{ }^{+}$pelas atividades de microorganismos. ${ }^{11}$ As concentrações de $\mathrm{NO}_{2}{ }^{-}$e $\mathrm{NO}_{3}{ }^{-}$foram negligenciáveis (juntos $<0,3 \%$ do NT), correspondendo em média (desvio padrão) a $0,006 \mathrm{mg} \mathrm{L}^{-1}\left(0,002 \mathrm{mg} \mathrm{L}^{-1}\right)$ e $0,10 \mathrm{mg} \mathrm{L}^{-1}(0,12$ $\left.\mathrm{mg} \mathrm{L}^{-1}\right)$, respectivamente.

\section{Eficiência da ETE e fatores de emissão de $\mathrm{N}_{2} \mathrm{O}$}

Os valores médios das eficiências de remoção de DBO (97\%), DQO (93\%) e NT (80\%) encontraram-se no limite superior das faixas reportadas por Oliveira e von Sperling, ${ }^{26}$ que apresentam uma análise de desempenho de 13 ETEs com sistemas de lodos ativados em operação nos estados de Minas Gerais e São Paulo. As faixas reportadas foram $74-96 \%$ para DBO, 62-93\% para DQO e $20-78 \%$ para NT. ${ }^{26}$ Oliveira e von Sperling ${ }^{26}$ ainda ressaltam que apenas 5 ETEs das 13 estudadas apresentaram eficiências de remoção de NT acima de $60 \%$.

O sistema de lodos ativados convencional empregado na ETE estudada proporciona a possibilidade de remoção biológica de nitrogênio (RBN). ${ }^{23}$ Entretanto, a emissão de $\mathrm{N}_{2} \mathrm{O}$ determinada durante $\mathrm{o}$ período de estudo, que representa em média $1,11 \mathrm{~kg} \mathrm{~N} \mathrm{dia}^{-1}$ e corresponde a $0,02 \%$ da carga de NT afluente à ETE, não explica a elevada remoção de NT atingida, necessitando de estudos posteriores para avaliação desse parâmetro. Ao contrário, a elevada eficiência de remoção de NT pode explicar as baixas emissões de $\mathrm{N}_{2} \mathrm{O}$ determinadas no presente estudo, uma vez que as concentrações de NT no efluente da ETE estudada sempre estiveram abaixo de $10 \mathrm{mg} \mathrm{N} \mathrm{L}^{-1}$, enquanto que, por exemplo, a ETE estudada por Brotto ${ }^{21}$ apresentou, no efluente, concentrações de NT sempre acima desse valor, com baixos níveis de remoção de $\mathrm{NT}$ e, consequentemente, emissões de $\mathrm{N}_{2} \mathrm{O}$ superiores ao presente estudo. Foley et al. ${ }^{27}$ concluíram, em estudo realizado em 7 ETEs projetadas para atender à RBN, que os processos de tratamento de esgotos que alcançam baixas concentrações de NT no efluente $(\leq$ $10 \mathrm{mg} \mathrm{N} \mathrm{L}^{-1}$ ) e, consequentemente, elevadas eficiências de remoção de NT, emitem menos $\mathrm{N}_{2} \mathrm{O}$ quando comparados a outros sistemas com baixos níveis de remoção de NT.
São poucos os trabalhos realizados com medições diretas de $\mathrm{N}_{2} \mathrm{O}$ em ETEs e, nestes, os FEs calculados com base na carga afluente de NT mostram grande variabilidade. ${ }^{11,12} \mathrm{~A}$ emissão de $\mathrm{N}_{2} \mathrm{O}$ na ETE estudada variou de $0,01 \%$ a $0,04 \%$ da carga de NT afluente. Essa faixa de emissão encontra-se no limite inferior dos FEs compilados no trabalho de revisão realizado por Law et al., ${ }^{11}$ que reúne dados de 11 estudos desenvolvidos em escala real (0-25\%) com diferentes processos de tratamento de esgotos, e também inferior ao valor proposto pelo IPCC ${ }^{9}(0,035 \%)$, assumindo uma carga de NT no esgoto bruto de $16 \mathrm{~g} \mathrm{~N}$ pessoa $^{-1} \mathrm{dia}^{-1}$ para países desenvolvidos. ${ }^{27}$

Os FEs médios de $\mathrm{N}_{2} \mathrm{O}$ obtidos no presente estudo, balizados na vazão de esgoto bruto, população atendida e carga de NT afluente encontrados, foram todos inferiores àqueles verificados em outros estudos realizados no Brasil (Tabela 1). Tal resultado pode ser explicado pelas diferentes condições empregadas nos processos de tratamento de esgotos de lodos ativados em cada ETE, ${ }^{27}$ onde a influência de alguns parâmetros operacionais, tais como concentração de NT afluente, concentração de OD, idade do lodo e o grau de aeração são os principais fatores de controle das emissões de $\mathrm{N}_{2} \mathrm{O}{ }^{11,12,20}$

Menores emissões de $\mathrm{N}_{2} \mathrm{O}$ são esperadas em sistemas de tratamento de esgotos que operam com idades do lodo altas e concentrações de OD superiores a $2 \mathrm{mg} \mathrm{L}^{-1}$, condições que favorecem o processo de nitrificação completo (i.e., $\mathrm{NH}_{4}{ }^{+} \rightarrow \mathrm{NO}_{2}{ }^{-} \rightarrow \mathrm{NO}_{3}{ }^{-}$). ${ }^{28,29}$ Da mesma forma, aqueles que operam com sistema anóxico a montante do tratamento aeróbio ( $\mathrm{OD} \leq 0,5 \mathrm{mg} \mathrm{L}^{-1}$ ), recirculação interna de nitratos e tempo de detenção hidráulica de 0,5 a 2,5 h, condições favoráveis ao processo de desnitrificação completo (i. e., $\mathrm{NO}_{3}{ }^{-} \rightarrow \mathrm{NO}_{2}{ }^{-} \rightarrow \mathrm{NO}$ $\rightarrow \mathrm{N}_{2} \mathrm{O} \rightarrow \mathrm{N}_{2}$ ). ${ }^{23}$ Tais processos proporcionam elevadas remoções de carga de NT e minimizam a geração de $\mathrm{N}_{2} \mathrm{O}$. O mesmo se verifica em sistemas que otimizam o grau de aeração empregado, uma vez que a injeção excessiva de ar nas zonas supersaturadas em $\mathrm{N}_{2} \mathrm{O}$ aceleram a transferência do gás para a atmosfera em virtude de uma maior turbulência do meio líquido. ${ }^{11,18,19}$

\section{Relação entre emissões de $\mathrm{N}_{2} \mathrm{O}$ e concentrações de $\mathrm{N}$ inorgânico no esgoto tratado}

A Figura 2 mostra que as emissões médias de $\mathrm{N}_{2} \mathrm{O}$ do tanque de aeração aumentam ao longo dos meses de amostragem, acompanhando a diminuição das concentrações de $\mathrm{NH}_{4}^{+}$e o crescimento do somatório das concentrações de $\mathrm{NO}_{2}{ }^{-}$e $\mathrm{NO}_{3}{ }^{-}$no efluente (Tabela 2). Esse comportamento sugere que as emissões médias de $\mathrm{N}_{2} \mathrm{O}$ estão associadas às maiores taxas de oxidação de $\mathrm{NH}_{4}^{+}$, corroborando com os resultados encontrados por Law et al. ${ }^{30}$ que encontraram através de observações obtidas em experimentos com variação nos níveis de $\mathrm{NH}_{4}^{+}$, OD e pH, em escala laboratorial, correlação exponencial entre as taxas de oxidação de $\mathrm{NH}_{4}{ }^{+}$e produção de $\mathrm{N}_{2} \mathrm{O}$.

As emissões de $\mathrm{N}_{2} \mathrm{O}$ mostram correlação negativa e estatisticamente significativa com as concentrações de $\mathrm{NH}_{4}{ }^{+}$no efluente $(\mathrm{r}=$ $0,95 ; \mathrm{n}=6 ; \mathrm{P}<0,01)$ e positiva e estatisticamente significativa $(\mathrm{r}=$ $0,91 ; \mathrm{n}=6 ; \mathrm{P}<0,01)$ com o somatório das concentrações de $\mathrm{NO}_{2}{ }^{-} \mathrm{e}$ $\mathrm{NO}_{3}{ }^{-}$no efluente. Tal resultado indica que a produção de $\mathrm{N}_{2} \mathrm{O}$ na ETE estudada possa estar relacionada ao processo de nitrificação, 
Tabela 2. Concentração dos compostos de nitrogênio ( \pm desvio padrão; $\mathrm{mg} \mathrm{L}^{-1}$ ) na saída (efluente) da ETE e o fluxo de $\mathrm{N}_{2} \mathrm{O}$ ( \pm desvio padrão; $\mathrm{g} \mathrm{N} \mathrm{m}^{-2}$ dia $^{-1}$ ) no tanque de aeração para cada dia de amostragem

\begin{tabular}{cccc}
\hline \multirow{2}{*}{$\begin{array}{c}\text { Amostragem } \\
(\text { dia/mês de 2010) }\end{array}$} & \multicolumn{2}{c}{ Saída da ETE $(\mathrm{n}=3)^{*}$} & $\begin{array}{c}\text { Tanque de aeração } \\
(\mathrm{n}=10)^{* *}\end{array}$ \\
\cline { 2 - 4 } & $\begin{array}{c}\mathrm{NH}_{4}^{+} \\
\left(\mathrm{mg} \mathrm{N} \mathrm{L}^{-1}\right)\end{array}$ & $\begin{array}{c}\mathrm{NO}_{2}^{-}+\mathrm{NO}_{3}{ }^{-} \\
\left(\mathrm{mg} \mathrm{N} \mathrm{L}^{-1}\right)\end{array}$ & $\begin{array}{c}\text { Fluxo de } \mathrm{N}_{2} \mathrm{O} \\
\left(\mathrm{g} \mathrm{N} \mathrm{m}^{-2} \mathrm{dia}^{-1}\right)\end{array}$ \\
\hline $14 / 01$ & $6,8 \pm 0,3$ & $0,62 \pm 0,03$ & $0,01 \pm 0,02$ \\
$09 / 02$ & $5,3 \pm 0,3$ & $1,29 \pm 0,06$ & $0,06 \pm 0,13$ \\
$12 / 03$ & $7,0 \pm 0,4$ & $0,99 \pm 0,05$ & $0,05 \pm 0,04$ \\
$30 / 04$ & $4,0 \pm 0,2$ & $1,32 \pm 0,07$ & $0,10 \pm 0,13$ \\
$18 / 05$ & $1,2 \pm 0,1$ & $2,69 \pm 0,13$ & $0,25 \pm 0,32$ \\
$29 / 06$ & $1,1 \pm 0,1$ & $1,45 \pm 0,07$ & $0,20 \pm 0,18$ \\
\hline
\end{tabular}

*Análises realizadas em triplicata. **Pontos de amostragem no tanque de aeração.

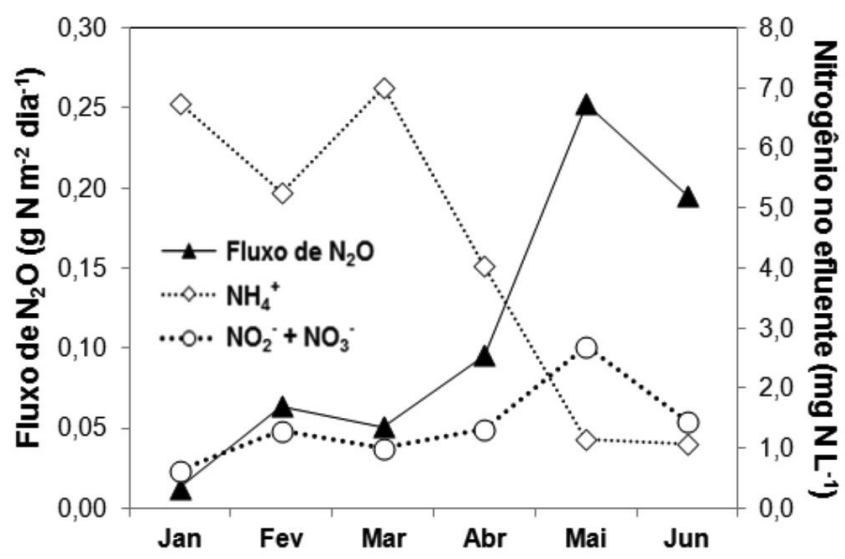

Figura 2. Variações das emissões médias de óxido nitroso, das concentrações de amônio e das concentrações de nitrito e nitrato juntos no efluente da ETE no período de janeiro a junho de 2010

no qual o $\mathrm{N}_{2} \mathrm{O}$ é produzido na etapa de oxidação de $\mathrm{NH}_{4}^{+}$a $\mathrm{NO}_{2}{ }^{-}$, mais precisamente, como um subproduto da oxidação de hidroxilamina $\left(\mathrm{NH}_{2} \mathrm{OH}\right)$ a $\mathrm{NO}_{2} \cdot{ }^{-14} \mathrm{O} \mathrm{N}_{2} \mathrm{O}$ ainda pode ser produzido através da decomposição química da $\mathrm{NH}_{2} \mathrm{OH}$. No entanto, esse mecanismo ainda é pouco esclarecido, sendo essa etapa do processo ainda bastante discutida na literatura. ${ }^{11,12,17,30} \mathrm{O} \mathrm{NO}_{2}^{-}$é posteriormente oxidado $\mathrm{NO}_{3}{ }^{-}$em condições de elevadas concentrações de OD ao final do processo. ${ }^{14}$

Segundo von Sperling, ${ }^{23}$ a taxa de crescimento das bactérias nitrificantes depende da disponibilidade de $\mathrm{NH}_{4}{ }^{+}$, onde a taxa de nitrificação pode ser expressa em termos das concentrações desse substrato, sendo a conversão de $\mathrm{NH}_{4}{ }^{+} \mathrm{a} \mathrm{N}_{2} \mathrm{O}$ influenciada por diversos parâmetros operacionais, tais como concentração de NT afluente, concentração de OD e idade do lodo. ${ }^{20}$ Esses parâmetros operacionais são diretamente responsáveis pela emissão de $\mathrm{N}_{2} \mathrm{O}$, além da intensidade da aeração do esgoto no tanque de aeração. ${ }^{11,18}$

\section{Relação entre emissões de $\mathrm{N}_{2} \mathrm{O}$ e concentrações de $\mathrm{NH}_{4}{ }^{+}$no esgoto afluente}

A emissão de $\mathrm{N}_{2} \mathrm{O}$ em processos de tratamento de esgotos é direta e indiretamente influenciada por alguns parâmetros operacionais, destacando-se dentre eles a concentração de NT afluente à ETE, principalmente na forma de $\mathrm{NH}_{4}{ }^{+}$. Esse parâmetro é variável ao longo do dia e pode influenciar diretamente nas emissões de $\mathrm{N}_{2} \mathrm{O} \cdot{ }^{19,20,22}$
Em estudo realizado em ETEs de lodos ativados nos EUA, Ahn et al. ${ }^{19}$ verificaram que a intensidade das emissões de $\mathrm{N}_{2} \mathrm{O}$ foi diretamente proporcional à carga de NT afluente. Em um estudo realizado ao longo de dois dias consecutivos, em escala piloto, Lotito et al. ${ }^{20}$ observaram que as maiores emissões de $\mathrm{N}_{2} \mathrm{O}$ do tanque de aeração ocorreram em resposta às maiores concentrações de $\mathrm{NH}_{4}^{+}$no esgoto afluente. Em estudo realizado durante seis dias consecutivos em uma ETE de lodos ativados com aeração intermitente, de Mello et $a l .{ }^{22}$ relataram significativa variação diária nas emissões de $\mathrm{N}_{2} \mathrm{O}$ e atribuíram principalmente à carga afluente de $\mathrm{NT}$, em especial $\mathrm{NH}_{4}{ }^{+}$.

A Figura 3a mostra correlação positiva e estatisticamente significativa entre as emissões médias de $\mathrm{N}_{2} \mathrm{O}$ e as concentrações de $\mathrm{NH}_{4}{ }^{+}$no afluente $(\mathrm{r}=0,90 ; \mathrm{n}=6 ; \mathrm{P}<0,01)$ ao longo do período de estudo. Tal resultado confirma a influência da concentração de NT afluente, principalmente na forma de $\mathrm{NH}_{4}^{+}$, na emissão de $\mathrm{N}_{2} \mathrm{O}$, corroborando com os resultados encontrados por Ahn et al.,${ }^{19}$ Lotito et al..$^{20}$ e de Mello et al..22
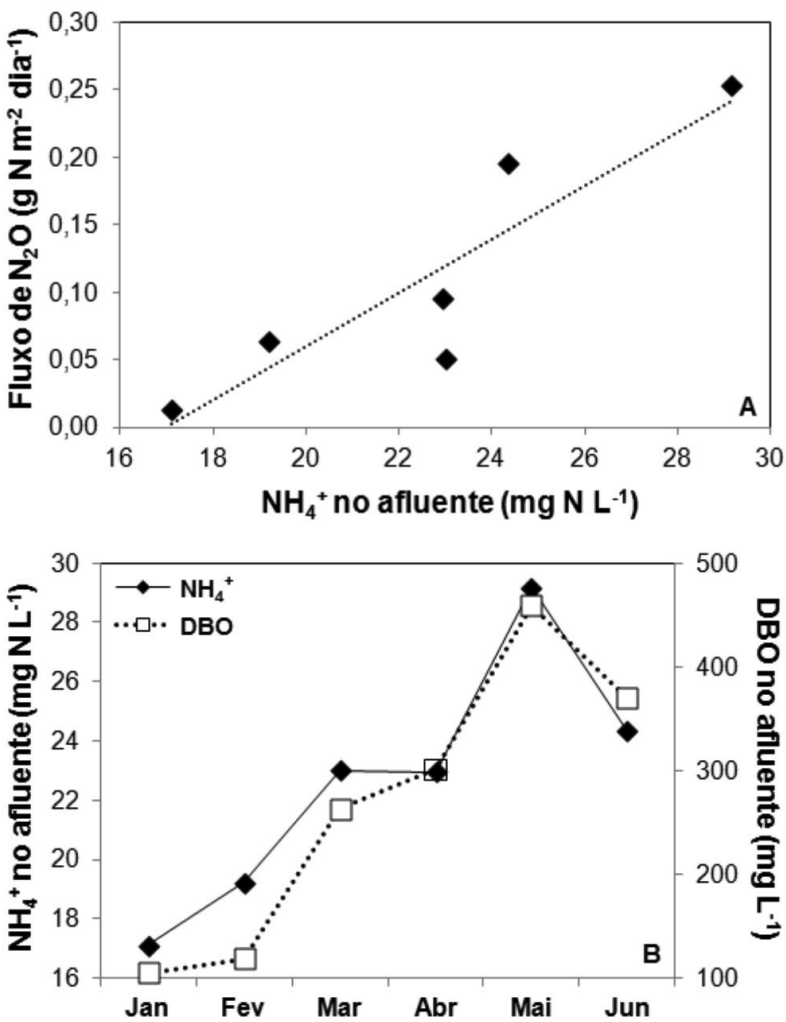

Figura 3. (a) Regressão linear entre as concentrações de amôniono afluente $e$ as emissões médias de óxido nitroso. (b) Variação das concentrações de amônio e demanda bioquímica de oxigênio afluente à ETE no período de janeiro a junho de 2010

A variação temporal das concentrações de $\mathrm{NH}_{4}{ }^{+}$no esgoto afluente da ETE estudada está relacionada com o maior uso da água nos meses de verão e/ou com a entrada de águas pluviais nas redes de coletas de esgoto, intensificando o efeito da diluição do esgoto bruto nessa época do ano, cenário característico de países de clima tropical. A Figura 3b mostra o comportamento sazonal das concentrações de $\mathrm{NH}_{4}{ }^{+}$e DBO no afluente, durante o período de estudo, confirmando esta hipótese (Tabela 3). De acordo com von Sperling, ${ }^{31}$ o consumo de água e a geração de esgoto em uma localidade variam ao longo do ano em decorrência de fatores climáticos. Climas mais quentes induzem a um maior consumo de água e, consequentemente, maiores vazões afluentes e menores concentrações de matéria orgânica e de NT. 
Tabela 3. Concentração de amônio e demanda bioquímica de oxigênio ( \pm desvio padrão; $\mathrm{mg} \mathrm{L}^{-1}$ ) na entrada (afluente) da ETE

\begin{tabular}{ccc}
\hline \multirow{2}{*}{$\begin{array}{c}\text { Amostragem } \\
(\text { mês de 2010) }\end{array}$} & \multicolumn{2}{c}{ Entrada da ETE } \\
\cline { 2 - 3 }$\left(\mathrm{mg} \mathrm{N} \mathrm{L}^{-1}\right)$ & $\begin{array}{c}\mathrm{DBO}^{+* *} \\
\left(\mathrm{mg} \mathrm{L}^{-1}\right)\end{array}$ \\
\hline Janeiro & $18,1 \pm 0,9$ & $105 \pm 9$ \\
Fevereiro & $20,3 \pm 1,0$ & $119 \pm 11$ \\
Março & $24,4 \pm 1,2$ & $263 \pm 24$ \\
Abril & $24,3 \pm 1,2$ & $301 \pm 27$ \\
Maio & $30,9 \pm 1,5$ & $459 \pm 41$ \\
Junho & $25,8 \pm 1,3$ & $370 \pm 33$ \\
\hline
\end{tabular}

*Análises realizadas em triplicata nas datas indicadas na Tabela 2. **Três determinações realizadas por mês por operadores da ETE.

\section{Relação entre emissões de $\mathrm{N}_{2} \mathrm{O}$ e concentrações de OD no tanque de aeração}

Das seis zonas que constituem o tanque de aeração, as que teoricamente operam com maior vazão de ar são a ZN2 $\left(6340 \mathrm{~m}^{3}\right.$ $\left.\mathrm{h}^{-1}\right)$ e ZN5 $\left(5700 \mathrm{~m}^{3} \mathrm{~h}^{-1}\right)$, pois são as que possuem o maior número de difusores de ar (Figura 1), enquanto que a ZN1 opera com baixa aeração $\left(140 \mathrm{~m}^{3} \mathrm{~h}^{-1}\right)$. Os fatores de controle das concentrações de OD são: a vazão de ar insuflado, o número de difusores presentes em cada zona e as taxas de oxidação da matéria orgânica e nitrificação pelas atividades microbiológicas.

A Figura 4a mostra o aumento gradual da concentração de OD da ZN1 até a ZN6, e o inverso em relação à DQO. Isso se explica em virtude da entrada do efluente do tratamento primário e do lodo
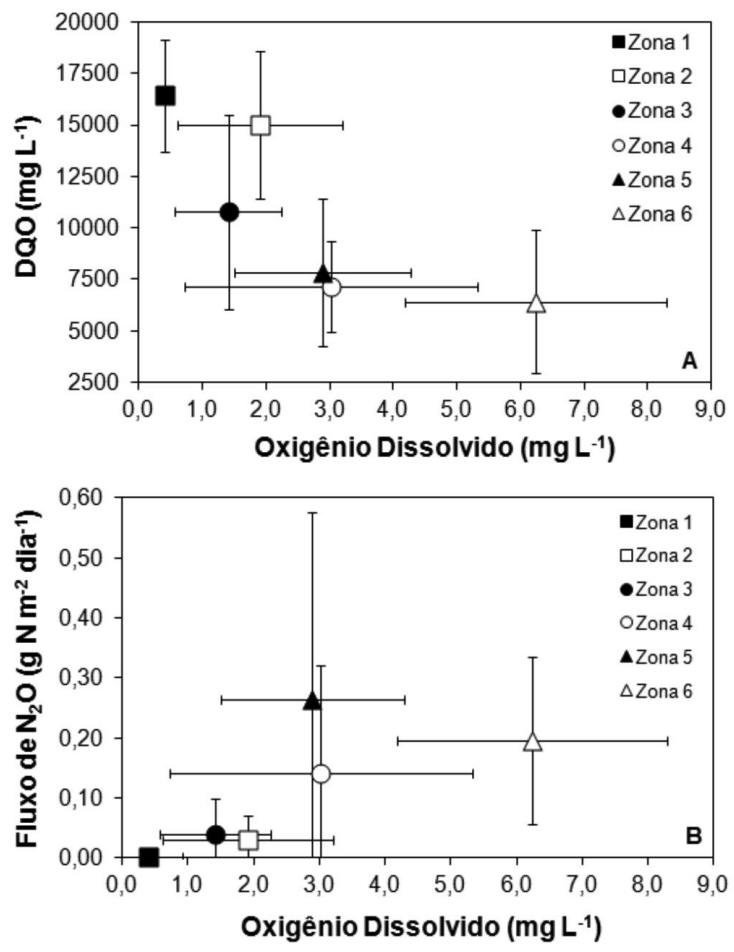

Figura 4. (a) Variação das concentrações médias da demanda química de oxigênio em função das concentrações médias de oxigênio dissolvido em 6 zonas dos 4 tanques de aeração. (b) Variação das emissões médias de óxido nitroso em função das concentrações médias de oxigênio dissolvido nas 6 zonas dos 4 tanques de aeração. As barras representam os desvios padrões das concentrações médias de 6 campanhas realizadas de janeiro a junho de 2010 biológico que retorna do decantador secundário ocorrer na ZN1, e o esgoto se deslocar desta em direção a ZN6 ao longo do processo de aeração diferenciado por zonas. Consequentemente, apesar de possuir um maior número de difusores em relação às demais, a ZN2 mostra baixa concentração de OD em virtude da grande carga orgânica que esta zona recebe de sua antecessora (ZN1). Já a ZN5, segunda zona em número de difusores, apresenta concentração de OD maior devido à gradual redução da carga orgânica ao longo das zonas, com disponibilidade de OD para ambas as reações de oxidação da matéria orgânica e nitrificação.

A Figura $4 b$ mostra que as maiores emissões de $\mathrm{N}_{2} \mathrm{O}$ ocorreram a partir da ZN4 onde as concentrações de OD foram superiores a 2 $m g L^{-1}$, condição favorável às maiores taxas de nitrificação $(\mathrm{OD} \geq 2$ mg L $\left.{ }^{-1}\right) .{ }^{32} \mathrm{~A}$ maior emissão média de $\mathrm{N}_{2} \mathrm{O}$ ocorreu na ZN5, onde a concentração média de $\mathrm{OD}$ foi $3 \mathrm{mg} \mathrm{L}^{-1}$. Esse resultado é próximo àquele encontrado por Brotto ${ }^{21}$ em uma ETE de lodos ativados, onde a emissão média de $\mathrm{N}_{2} \mathrm{O}$ foi aproximadamente seis vezes superior à encontrada no presente estudo. O processo estudado por Brotto ${ }^{21}$ é classificado como de aeração modificada, em virtude da baixa idade do lodo adotada ( $c a .3$ dias), quatro vezes inferior à idade do lodo da ETE do presente estudo, o que favorece o processo de nitrificação apenas parcial, ou seja, a etapa de oxidação de $\mathrm{NH}_{4}{ }^{+}$a $\mathrm{NO}_{2} \cdot{ }^{-33} \mathrm{Os}$ processos de tratamento de esgotos que utilizam a aeração como método de oxidação da matéria orgânica devem evitar a ocorrência de nitrificação parcial ou intermitente, minimizando, dessa forma, as emissões de $\mathrm{N}_{2} \mathrm{O} .{ }^{19}$

\section{CONCLUSÕES}

Os resultados mostram que o processo de tratamento de esgotos por lodos ativados convencional empregado na ETE estudada é bastante eficiente no que tange a remoção de carbono orgânico e NT. Além disso, apresenta baixas emissões de $\mathrm{N}_{2} \mathrm{O}$, correspondendo em média a $0,02 \%$ da carga de NT afluente. Sugere-se que tal eficiência esteja relacionada à utilização de aeração suficiente para promover condições favoráveis aos processos microbiológicos de oxidação da matéria orgânica e ao processo de nitrificação completo mesmo nas condições de elevadas concentrações de DBO, DQO e de NT (especialmente $\mathrm{NH}_{4}^{+}$), como observados no período de inverno.

A ETE estudada foi a que apresentou os menores FEs médios, balizados na vazão de esgoto, população atendida e carga de NT afluente, em relação àqueles relatados em estudos desenvolvidos no exterior ${ }^{11,12,19,27}$ e no Brasil, ${ }^{18,21,22}$ bem como em relação aos propostos pelo IPCC ${ }^{9}$ e US-EPA. ${ }^{10}$ Apesar de a ETE não possuir uma unidade de tratamento terciário, a eficiência alcançada na remoção de NT sugere atuação semelhante às verificadas em ETEs com RBN. Consequentemente, menores concentrações de NT no efluente final são alcançadas, o que deve explicar também as menores emissões de $\mathrm{N}_{2} \mathrm{O}$ observadas nesse estudo quando comparadas às emissões em outras ETEs já estudadas no Brasil. ${ }^{18,21,22}$ Além disso, a menor abundância de $\mathrm{N}$ no efluente minimiza posteriores emissões de $\mathrm{N}_{2} \mathrm{O}$ nos corpos hídricos receptores (e.g., rios, estuários e águas costeiras).

\section{AGRADECIMENTOS}

Este estudo teve apoio do PAPES-Fiocruz/CNPq (Proc.: 0477 - 2008/2011) e da FAPERJ (Proc.: E-26/112.140/2008). Provimento de bolsas: W. Z. de Mello (CNPq, PQ, Proc.: 3014212009/9), R. B. Alvim (CNPq, mestrado, Proc.: 147848/2010-5), R. P. Ribeiro (FAPERJ (Programa Bolsa Nota 10), mestrado, Proc.: E-26/100.276/2012) e S. A. Andrade (UFF/CNPq, IC). 


\section{REFERÊNCIAS}

1. IPCC, Intergovernmental Panel on Climate Change; Climate Change 2001: The Scientific Basis: Contribution of Working Group I to the Third Assessment Report of the Intergovernmental Panel on Climate Change, New York, 2001.

2. Crutzen, P. J.; Annu. Rev. Earth Planet. Sci. 1979, 7, 443.

3. Abbat, J. P. D.; Molina, M. J.; Annu. Rev. Energy Environ. 1993, $18,1$.

4. Ravishankara, A. R.; Daniel, J. S.; Portman, R. W.; Science 2009, 326, 123.

5. IPCC, Intergovernmental Panel on Climate Change; Climate Change 2007: The Physical Science Basis - Contribution of Working Group I to the Fourth Assessment Report of the Intergovernmental Panel on Climate Change, Cambridge, 2007.

6. Syakila, A.; Kroeze, C.; Greenhouse Gas Measurement \& Management 2011, $1,17$.

7. http://www.esrl.noaa.gov/gmd/hats/combined/N2O.html, acessada em Junho 2012.

8. Fluckiger, J.; Dallenbach, A.; Blunier, T.; Stauffer, B.; Stocker, T. F.; Raynaud, D.; Barnola, J. M.; Science 1999, 285, 227.

9. IPCC, Intergovernmental Panel on Climate Change; 2006 IPCC Guidelines for National Greenhouse Gas Inventories, Waste - Vol. 5, Kanagawa, 2006.

10. U.S. EPA, United States Environmental Protection Agency; Inventory of U.S. Greenhouse Gas Emissions and Sinks: 1990-2009, Washington, 2011.

11. Law, Y.; Ye, L.; Pan, Y.; Yuan, Z.; Phil. Trans. R. Soc. B 2012, 367, 1265.

12. Kampschreur, M. J.; Temmink, H.; Kleerebezem, R.; Jetten, M. S. M.; van Loosdrecht, M. C. M.; Water Res. 2009, 43, 4093.

13. Czepiel, P.; Crill, P.; Harriss, R.; Environ. Sci. Technol. 1995, $29,2352$.

14. Wrage, N.; Velthof, G. L.; van Beusichem, M .L.; Oenema, O.; Soil Biol. Biochem. 2001, 33, 1723.

15. Rassamee, V.; Sattayatewa, C.; Pagilla, K.; Chandran, K.; Biotechnol. Bioeng. 2011, 108, 2036.

16. Kim, S. W.; Miyahara. M.; Fushinobu, S.; Wakagi, T.; Shoun, H.; Bioresour. Technol. 2010, 101, 3958.
17. Wunderlin, P.; Mohn, J.; Joss, A.; Emmenegger, L.; Siegrist, H.; Water Res. 2012, 46, 1027.

18. Brotto, A. C.; Kligerman, D. C.; Piccoli, A. S.; de Mello, W. Z.; Quim. Nova 2010, 33, 618 .

19. Ahn, J. O.; Kim, S.; Park, H.; Rahm, B.; Pagilla, K.; Chandran, K.; Environ. Sci. Technol. 2010, 44, 4505.

20. Lotito, A. M.; Wunderlin, P.; Joss, A.; Kipf, M.; Siegrist, H.; Water Res. 2012, 46, 3563 .

21. Brotto, A. C.; Dissertação de Mestrado, Universidade Federal Fluminense, Brasil, 2011.

22. de Mello, W. Z.; Kligerman, D. C.; Picolli, A. S.; Ribeiro, R. P.; Oliveira, J. L. M.; Brotto, A. C.; Quim. Nova 2013, 36, 16.

23. von Sperling, M. Lodos ativados, vol. 4, Princípios do Tratamento Biológico de Águas Residuárias, 2 ed., Departamento de Engenharia Sanitária e Ambiental da Universidade Federal de Minas Gerais: Belo Horizonte, 2002.

24. Eaton, A. D.; Clesceri, L. S.; Greenberg, A. E.; Standard Methods for the Examination of Water and Wastewater, 21 ed., American Public Health Association: Washington, 2005.

25. Jordão, E. P.; Pessôa, C. A. Tratamento de Esgotos Domésticos, SEGRAC: Rio de Janeiro, 2005.

26. Oliveira, S. M. A. C.; von Sperling, M.; Eng. Sanit. Ambient. 2005, 10, 347.

27. Foley, J.; de Hass, D.; Yuan, Z.; Lant, P.; Water Res. 2010, 44, 831.

28. Noda, N.; Kaneko, N.; Mikami, M.; Kimochi, Y.; Tsuneda, S.; Hirata, A.; Mizuochi, M.; Inamori, Y.; Water Sci. Technol. 2003, 48, 363.

29. Tallec, G.; Garnier, J.; Billen, G.; Gousailles, M.; Water Res. 2006, 40, 2972.

30. Law, Y.; Ni, B. J.; Lant, P., Yuan, Z.; Water Res. 2012, 46, 3409.

31. von Sperling, M. Introdução a Qualidade das Águas e ao Tratamento de Esgoto, vol. 1, Princípios do Tratamento Biológico de Águas Residuárias, 3 ed., Departamento de Engenharia Sanitária e Ambiental da Universidade Federal de Minas Gerais: Belo Horizonte, 2005.

32. Surampalli, R. Y.; Tyagi, R. D.; Scheible, O. K.; Heidman, J. A.; Bioresour. Technol. 1997, 61, 151.

33. Pollice A.; Tandoi, V.; Lestingi, C.; Water Res. 2002, 36, 2541 